\title{
An unlevel playing field
}

To the Editor - I would like to reflect on one sentence "if a lab is in competition with many other groups who have similar technical capabilities, they may not want to give up any small advantage that they have" in your editorial entitled 'A problem shared is a problem halved' (Nat. Phys. 15, 107; 2019).

I would refine this statement because I believe that the word "similar" is not appropriate. Here in Hungary, science has poor funding with respect to other European countries, even when compared to the country's gross domestic product (not to mention working conditions, stability and teaching duties). Nevertheless, to publish in a high-impact journal or to get EU funding, we are supposed to compete with large, well-funded and often technology-oriented German and Swiss groups. In these large groups, it only takes a short time to pick up a good idea, put two post-docs and two students on it, and finish off by publishing a number of papers on the subject, gradually forgetting to cite the original work, and then even getting the credit for the technological development. In the case of a code, such as the density matrix renormalization group (DMRG) package developed by Örs Legeza from the Wigner Institute of the Hungarian Academy of Sciences, or the non-Abelian MPO code of my student, Miklós Werner, the development can take 3,5 or even 10 years of dedicated work. These codes are unique, and they can solve problems that no one else can, at least for a few years.

And this is the only way we can remain in competition. Code sharing would remove the last barriers in this process.

I should add that I am not really against open-source codes. In fact, we did make some of our codes accessible to the public (see, for example, the Budapest DM-NRG code, http://www.phy.bme.hu/ dmnrg/). But, as also explained in the editorial, making a code public such that it is useful is a major effort. In fact, although we planned to initially, we never posted the beta version of this open-access DM-NRG code because we just did not have the human resources for writing the manuals, building the web page and to do all the necessary work to make a code public. And human resources is a pure question of money, according to our experience. Therefore, even on the 'scientific market' of open-access codes, being well funded is an extreme advantage.

All in all, I believe that an open-source code policy would work similarly to the free market at the European or US level: the overall 'production' may indeed increase but the resulting goods will be distributed quite unevenly, states already in a good position would mostly benefit from it while local 'production' in poorer states would suffer and eventually disappear.

I personally believe that code sharing should be the personal decision of those who develop the code.

\section{Gergely Zaránd}

Institute of Physics, Budapest University of

Technology and Economics, Budapest, Hungary.

e-mail:zarand@eik.bme.hu

Published online: 1 August 2019

https://doi.org/10.1038/s41567-019-0619-0

\section{Credit and incentives}

To the Editor - It is obvious that data transparency and the availability of raw experimental data must be the future standard practice in the experimental sciences. While as an experimentalist I am barely affected by it, I do have reservations against forcing our colleagues in the numerical theoretical sciences to make all code available publicly, a push mainly enforced by funding agencies. This treats the experimental and theoretical sciences on an unequal footing, and in the long run may hurt the advancement of numerical science.

It is common practice to develop experimental capabilities in a laboratory and perfect it over many years. The effort to achieve superconductivity of sulfur hydride under extreme pressures is a good example of this. Despite the open publication of the design of the apparatus, typically the know-how gives those who develop it a competitive advantage to drive the science.

The situation in the theoretical sciences is completely analogous. Hard problems are tackled and advances in concepts, algorithms and computational means expand our understanding of physical systems. Yet, simply implementing policies enforcing such a 'digital apparatus' to be made available immediately will be detrimental to the development efforts, jeopardize the careers of young scientists developing such code, and most importantly will miss the opportunity to encourage code sharing in a way that is constructive for those who wrote it.

We as a community have to shape the cultural change towards completely open data and code. Critically, we have to redefine the way how credit is assigned for developmental efforts. Instead of forced publication, I believe that positive incentives are a more proper way to fairly ensure the sharing of code. Part of this lies with the prominent publishing houses. Will the Nature journals publish prominently released code on its own, separate from the scientific discoveries made by it?

Philip J. W. Moll(D)

Institute of Materials, École Polytechnique Fédérale de Lausanne, Lausanne, Switzerland.

e-mail:philip.moll@epfl.ch

Published online: 22 July 2019

https://doi.org/10.1038/s41567-019-0623-4 